\title{
Quantitative measurements of HER2 and phospho-HER2 expression: correlation with pathologic response to neoadjuvant chemotherapy and trastuzumab
}

Huan Cheng ${ }^{1}$, Yalai Bai ${ }^{1}$, William Sikov², Natalie Sinclair ${ }^{2}$, Veerle Bossuyt ${ }^{1}$, Maysa M Abu-Khalaff, Lyndsay N Harris ${ }^{4}$ and David L Rimm ${ }^{1 *}$

\begin{abstract}
Background: Preoperative therapy with chemotherapy and the HER2-targeted monoclonal antibody trastuzumab is valuable for patients with large or locally advanced HER2-positive (HER2+) breast cancers but traditional methods of measuring HER2 expression do not accurately stratify patients for likelihood of response. Quantitative immunofluorescent approaches have the potential to provide a mathematically continuous measure of HER2. Here we seek to determine whether quantitative measurement of HER2 or phospho-HER2 correlates with likelihood of response to trastuzumab- containing neoadjuvant therapy.

Methods: We evaluated core biopsy samples from 27 HER2+ breast cancer patients enrolled in a preoperative clinical trial using trastuzumab, nab-paclitaxel and carboplatin combination therapy (BrUOG BR-211B (NCT00617942)). Tumor core biopsies were taken before initiation of treatment and 9-13 days after patients received "run-in" doses of either single agent trastuzumab or nab-paclitaxel. The AQUA method of quantitative immunofluorescence was used for analysis of in situ protein expression. Patients then received 18 weeks of treatment, followed by surgery to assess pathologic response to the neoadjuvant regimen.
\end{abstract}

Results: A HER2 score of 2111 by AQUA analysis has been shown to be equivalent to HER2 3+ by immunohistochemical staining in previous studies. Of 20 evaluable patients, 10 cases who achieved a pathologic complete response (pathCR) with neoadjuvant treatment had a mean HER2 level of 10251 compared with 4766 in the patients without pathCR $(p=0.0021)$. Measurement of phospho-HER2 showed no difference in pathCR vs non-pathCR groups. In 9 patients who had HER2 levels repeated after a single treatment with trastuzumab there was no evidence of a reduction in the HER2 or phospho-HER2 levels following that exposure.

Conclusions: High levels of HER2 are associated with achievement of a pathCR in the preoperative setting, while levels of Phospho-HER2 were not predictive of response. This data suggests that accurate measurement of HER2 may help determine the likelihood of response in the pre-surgical setting. Further validation in larger cohorts is required, but this pilot data shows the feasibility of this approach.

Keywords: Immunohistochemistry, Immunofluorescence

\footnotetext{
* Correspondence: David.Rimm@yale.edu

'Department of Pathology, Yale University School of Medicine, 310 Cedar

Street, PO Box 208023, New Haven, CT 06520-8023, USA

Full list of author information is available at the end of the article
} 


\section{Background}

Human epidermal growth factor receptor 2 (HER2) is amplified or over-expressed in around $20 \%$ of breast cancer cases, and the amplification of HER2 is usually associated with worse prognosis [1-3]. Trastuzumab, a humanized monoclonal antibody, was the first drug developed to target HER2 amplified breast cancer. The addition of trastuzumab to cytotoxic chemotherapy showed significant improved time to progression, overall response rate, response duration and overall survival (OS) in advanced HER2-positive breast cancer (HER2+), resulting in FDA approval of the drug in 1998 [4]. In 2006, FDA approval was extended to use of the drug in combination with chemotherapy in the adjuvant setting in early stage HER2 positive breast cancer $[5,6]$. There are several proposed mechanisms of action. Some studies suggest that the drug disrupts ligandindependent transmembrane signaling triggered by the formation of HER2:HER2 homodimers or HER2:HER3 heterodimers, thereby diminishing Akt pathway activation, which ultimately leads to cell apoptosis [7]. Other mechanisms of cytotoxicity, including activation of antibodydependent cell-mediated cytotoxicity (ADCC) [8] or blocking the cleavage of HER2 extracellular domain [9] have also been described.

Pre-surgical or neoadjuvant chemotherapy is standard therapy for inflammatory and locally advanced breast cancer. The addition of trastuzumab to chemotherapy in the pre-surgical setting in HER2+ patients has been tested in several phase II studies [10-14], with pathological complete response (pathCR) rates ranging from $18 \%$ to $47 \%$. A phase II-III randomized pre-surgical trial conducted by the M.D. Anderson Cancer Center shows significant improvement in the pathCR rate with the addition of trastuzumab to chemotherapy [15]. The NOAH (NeO-Adjuvant Herceptin) trial is a phase III trial that evaluated the addition of trastuzumab to anthracyclineand taxane -based chemotherapy for HER2-positive patients with locally advanced or inflammatory breast cancer. Again, the addition of trastuzumab resulted in an increased pathCR rate, which translated into improved event free survival and OS [16]. In the phase III GeparQuattro trial, evaluating the effect of the addition of capecitabine to epribubin/cyclophosphamide/docetaxel regime, 445 HER2+ patients among 1509 patients with operable or locally advanced tumors were also given trastuzumab. The pathCR rate in the HER2+ subset was $31.7 \%$ [17]. Taken together, these trials suggest that the addition of trastuzumab to pre-surgical chemotherapy significantly improves outcomes in HER $2+$ breast cancer patients.

Although the use of trastuzumab as part of the presurgical regimen for breast cancer has increased, a uniform clinical benefit of trastuzumab in combination with chemotherapy is not observed. The likelihood of achieving a pathCR in this cohort is higher in trastuzumab treated patients with hormone receptor- negative HER2+ cancers compared to hormone receptor-positive HER2+ cancers [18]. Other groups have used mRNA measurements to predict pathCR. For example Denkert and colleagues found that quantitative assessment of mRNA for ESR1 and HER2 can predict pathCR [19]. Still, there remains no uniformly accepted method to predict which HER2+ patients are more or less likely to achieve a pathCR. Currently the only standard diagnostic tests to support the addition of trastuzumab are IHC and FISH and neither predicts pathological complete response. As treatment options expand for treatment of HER2 driven tumors, increased assay predictability and specificity will be sought. Here, we seek to identify quantitative measurement of HER2 protein level that are able to specifically select the patients who will most benefit from a trastuzumab containing treatment regime, and those who are not likely to achieve the desired pathCR status.

BrUOG BR-211B (NCT00617942) was a pre-surgical trial for stage II-III HER2+ breast cancer patients led by the Brown University Oncology Group (BrUOG) and the Yale Cancer Center. Patients were treated with q3week carboplatin, weekly nab-paclitaxel and trastuzumab for 18 weeks. In this trial, research biopsies were collected before initiation of treatment and again after a brief "run-in" exposure to either trastuzumab or nab-paclitaxel before patients received their first doses of the entire treatment regimen, allowing the testing of two hypotheses: 1) that the level of HER2 or pHER2 is associated with the likelihood of achieving a pathological complete response; and 2) that short exposure "run-in" treatment with trastuzumab alters the expression of HER2 or pHER2. In this paper, we quantitatively measured the level of HER2 and pHER2 (pY1248) to address these two issues.

\section{Methods}

\section{BrUOG BR-211B study design}

The trial, BrUOG BR-211B (NCT00617942, initiated February 6, 2008), was designed to determine the clinical and pathologic response rates of treatment with q3week carboplatin, weekly nab-paclitaxel and weekly trastuzumab in resectable and unresectable locally advanced breast cancer. Eligibility for the trial included histologically documented adenocarcinoma, female age greater than 18, stage IIA-IIIC disease, no evidence of metastatic disease, no prior systemic therapy, not pregnant or lactating, no baseline neuropathy greater than or equal to grade 2, and HER2 positive defined by IHC $3+$ or FISH ratio greater than or equal to 2.0. Baseline biopsies were obtained before the treatment. Randomized "run-in" treatment was given to patients with either a single dose of trastuzumab $6 \mathrm{mg} / \mathrm{kg}$ or two weekly doses of nab- 
paclitaxel $100 \mathrm{mg} / \mathrm{m}^{2}$ (determined by the institution at which the patient enrolled on the study). After 9 13 days biopsies were repeated. Patients then received trastuzumab $2 \mathrm{mg} / \mathrm{kg}$ weekly (patients who received "run-in" nab-paclitaxel were treated with $4 \mathrm{mg} / \mathrm{kg}$ for the first week), weekly nab-paclitaxel $100 \mathrm{mg} / \mathrm{m} 2$ and carboplatin AUC 6 every 3 weeks for 18 weeks. Pathologic complete response (defined as the absence of residual invasive breast cancer in the breast and axillary nodes) was then assessed at definitive surgery within 6 weeks from the last dose of pre-surgical therapy. Pairs of biopsies (before and after "run-in" treatment) from twenty-seven patients were obtained for biomarker studies (Tables 1 and 2). This trial received ethical approval from both the Yale Human Investigation Committee and the institutional review boards at the participating BrUOG hospitals (Women and Infants Hospital, Rhode Island Hospital, Miriam Hospital, Roger Williams Medical Center and Memorial Hospital of Rhode Island). These review boards approved the consent forms and consent was obtained from each patient prior to enrolling in the trial.

\section{Immunofluorescence and AQUA analysis of tissue biopsies}

Formalin fixed paraffin embedded tissue biopsies from twenty-seven patients were collected and immediately fixed in formalin to prevent artifact associated with delayed time to fixation. Specimens were then processed, sectioned and deparaffinized in xylene, followed by antigen retrieval at $97^{\circ} \mathrm{C}$ in $\mathrm{pH} 6$ sodium citric buffer for 20 minutes. Slides were incubated with $0.3 \%$ bovine serum albumin in $0.1 \mathrm{M}$ tris-buffered saline with $0.05 \%$

\section{Table 1 Cohort description}

\begin{tabular}{lll}
\hline Variables & $\mathbf{n}$ & $\%$ \\
\hline ER & 14 & $51.9 \%$ \\
Negative & 9 & $33.3 \%$ \\
Positive & 4 & $14.8 \%$ \\
Unknown & & \\
PR & 18 & $66.7 \%$ \\
Negative & 5 & $18.5 \%$ \\
Positive & 4 & $14.8 \%$ \\
Unknown & & \\
Run-in Treatment & 17 & $63.0 \%$ \\
Trastuzumab & 10 & $37.0 \%$ \\
Nab-paclitaxel & & \\
Response & 13 & $48.1 \%$ \\
PathCR & 10 & $37.0 \%$ \\
No PathCR & 4 & $14.8 \%$ \\
Unknown & &
\end{tabular}

Table 2 Association with PathCR

\begin{tabular}{lllll}
\hline Variable & PathCR & No PathCR & Chi-Sq & p \\
\hline ER & & & 0.833 & 0.3613 \\
Negative & $7(35.0 \%)$ & $5(25.0 \%)$ & & \\
Positive & $3(15.0 \%)$ & $5(25.0 \%)$ & & \\
PR & & & 5.000 & 0.0253 \\
Negative & $10(50.0 \%)$ & $6(30.0 \%)$ & & \\
Positive & $0(0.0 \%)$ & $4(20.0 \%)$ & & \\
Run-in Treatment & & & 1.308 & 0.2528 \\
Nab-paclitaxel & $7(30.4 \%)$ & $3(13.1 \%)$ & & \\
Trastuzumab & $6(26.1 \%)$ & $7(30.4 \%)$ & & \\
\hline
\end{tabular}

Tween-20 to block non-specific binding, then incubated with primary antibodies at $4 \mathrm{C}$ overnight. The primary antibodies to HER2 (CB11, Biocare, Concord, MA) and to phospho-HER2 pY1248 (PN2A, LabVision, Fremont, $\mathrm{CA}$ ) have been previously extensively validated and were only tested for reproducibility before use in our studies. Antibodies diluted 1:1000 and 1:100 respectively showed uniform membrane staining as has been previously described for each (Figure 1). Then the slides were treated with Mouse Envision" (Dako, Carpinteria, CA) at room temperature for one hour. Cy5 tyramide (NEN Life Science Products, Boston, MA) was then incubated for $10 \mathrm{~min}$ at room temperature. Cytokeratin was stained by polyclonal rabbit anti-pan cytokeration (Dako, Carpinteria, $\mathrm{CA}$ ) and DAPI was then added to define tumor region and subcellular localization required for AQUA analysis.
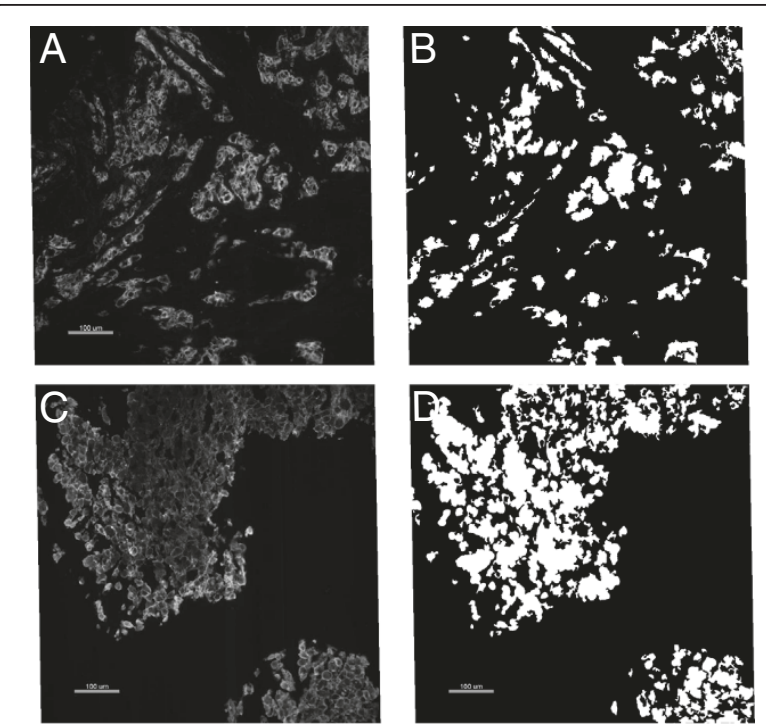

Figure 1 HER2 and pHER2 staining. A: A representative image of HER2 expression visualized with CB11 antibody; B: Tumor area defined by cytokeratin expression for image $\mathbf{A}$; $\mathbf{C}$ : phopho HER2 (pY1248) expression visualized with the PN2A antibody; D: Tumor area defined by cytokeratin for image $\mathbf{C}$. 
The algorithm of AQUA analysis is described in detail elsewhere [20,21]. Briefly, regions that contain positive cytokeratin signal were defined as region of interest (ROI), signal of total intensity of a target in each ROI was measured under tumor mask defined by cytokeratin staining and normalized by light source, filter and exposure time, then the total intensity is divided by tumor mask area to generate an AQUA score of each ROI, which is a quantitative measure of the target protein normalized by tumor area. AQUA scores from all of the ROI from each biopsy sample were then averaged to represent the AQUA score for the biopsy.

\section{Statistical analysis}

The statistical calculations were performed using StatView (SAS, Cary, NC). Pathological complete response was used to stratify patients, Fischer's PLSD was used to compare the mean difference of target biomarkers within each groups. Ordinary least square (OLS) method and paired t-test were used to test if run-in treatment alternated the expression of biomarkers.

\section{Results}

\section{Association of HER2 level before and after one dose treatment with trastuzumab response}

Among the 27 patients, assessment of pathologic response was available for 23 . Of these patients, 20 pretreatment biopsies were evaluable, as were 12 of the repeat biopsies after 'run-in' treatment. Among the 20 patients with evaluable tissue and known response, 50\% [10] had pathCR. The mean baseline HER2 level measured by CB11 antibody in the pathCR group is 10214 AQUA score units, compared with 4766.3 in the no pathCR group $(\mathrm{p}=0.0021)$ (Table 3$)$. This result suggests that a higher HER2 level is associated with pathological complete response. In other studies, we have shown that the lowest AQUA score in a patient with a cancer that was HER2 $3+$ by immunohistochemical staining was 2111.6 (data not shown) [22] and therefore the mean of HER2 expression in the group with no pathCR is well above the minimum HER2 3+ level. While there was not a substantial change in HER2 levels between the baseline and post-'run-in' samples in either group, due to the small number of post-'run-in' biopsy samples in the pathCR group the difference in HER2 levels between the pathCR and no pathCR groups for that timepoint no longer reached statistical significance (Table 3).

Since there is a known inverse association between estrogen receptor (ER) and response in the pre-surgical setting, we evaluated the mean levels of HER2 protein as a function of clinical ER status. The mean AQUA score in ER negative patients was 8615 compared to the 5498 in ER positive patients. This difference is not statistically
Table 3 Association between the level of HER2 and PathCR

\begin{tabular}{|c|c|c|c|c|c|c|c|}
\hline \multicolumn{8}{|c|}{ A } \\
\hline & \multicolumn{3}{|c|}{ no-PathCR } & \multicolumn{3}{|c|}{ PathCR } & \multirow[b]{2}{*}{$P$ value } \\
\hline & $\mathbf{N}$ & Mean & SEM & $\mathrm{N}$ & Mean & SEM & \\
\hline $\begin{array}{l}\text { HER2 AQUA } \\
\text { (base) }\end{array}$ & 10 & 4766.3 & 1381.1 & 10 & 10214.6 & 639.1 & 0.0021 \\
\hline $\begin{array}{l}\text { HER2 AQUA } \\
\text { (post) }\end{array}$ & 9 & 4853.5 & 1428.0 & 3 & 8309.3 & 1256.0 & 0.2191 \\
\hline $\begin{array}{l}\text { Post/Base } \\
\text { AQUA ratio }\end{array}$ & 9 & 1.09 & 0.203 & 2 & 0.765 & 0.089 & 0.4897 \\
\hline \multicolumn{8}{|c|}{ B } \\
\hline & \multicolumn{3}{|c|}{ no-Path CR } & \multicolumn{3}{|c|}{ Path CR } & \\
\hline & $\mathbf{N}$ & Mean & SEM & $\mathrm{N}$ & Mean & SEM & $P$ value \\
\hline $\begin{array}{c}\text { pHER2 AQUA } \\
\text { (base) }\end{array}$ & 10 & 2183.7 & 472.2 & 11 & 2604.9 & 538.7 & 0.5669 \\
\hline $\begin{array}{l}\text { pHER2 AQUA } \\
\text { (post) }\end{array}$ & 9 & 2482.7 & 507.2 & 7 & 3042.4 & 1005.5 & 0.6030 \\
\hline $\begin{array}{l}\text { Post/Base } \\
\text { AQUA ratio }\end{array}$ & 9 & 1.49 & 0.326 & 6 & 0.919 & 0.145 & 0.1953 \\
\hline
\end{tabular}

significant in a two sample mean test $(\mathrm{p}=0.1070$, Figure 2$)$ in our limited patient population.

Among the 27 patients, 17 were treated with trastuzumab as the "run-in" treatment and 10 were treated with nab-paclitaxel. Nine of 17 trastuzumab treated patients had paired evaluable baseline and post-treatment biopsies. We compared the HER2 level in these 9 pairs. Linear regression with fixed intercept term showed an $R^{2}$ value of 0.83 and a slope term of 0.93 (post-treatment AQUA score unit as the dependent variable). This slope is not statistically significantly different from 1, suggesting that HER2 level is not affected by a single dose of trastuzumab treatment. The result is confirmed by the paired t-test of these 9 pairs of baseline and post-treatment biopsies - the mean of post baseline difference is 449.4 AQUA score units $(\mathrm{p}=0.4181)$ (Figure 3A).

\section{Association of phospho-HER2 (pY1248) level before and} after one dose treatment with response to trastuzumab For the phospho-HER2 studies, 21 out of 23 biopsies before single dose treatment were evaluable. Also 17 out of 23 biopsies after one dose run-in treatment were evaluable. Among the 21 patients with evaluable tissue and annotated response, 9 had a PathCR (47.3\%). The mean of pHER2 level measured by the PN2A antibody in the pathological complete response group was 2768.0 AQUA score units, compared with 2183.7 in the no PathCR group $(\mathrm{p}=0.4689)$ (Table 3$)$. This result suggested that pHER2 level was not associated with pathological complete response. We also tested the mean of pHER2 level after one dose therapy or the pHER2 ratio between posttreatment and baseline biopsies, neither of which was associated with pathological complete response (Table 3). 


\section{HER2 AQUA score vs ER status}

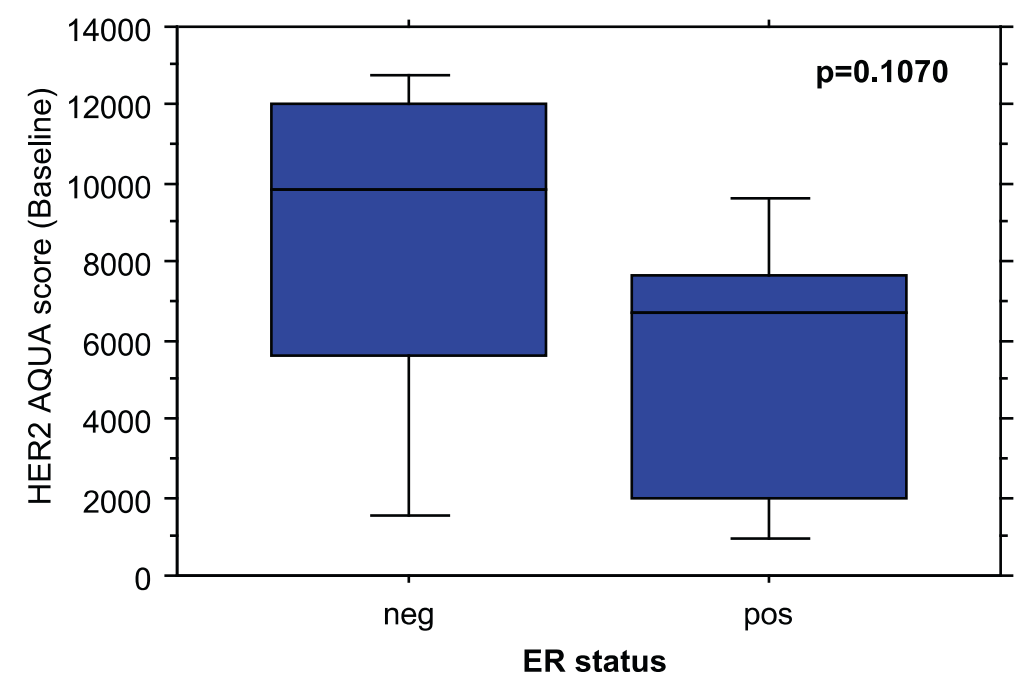

Figure 2 HER2 AQUA score is not statistically significantly correlated with ER status.

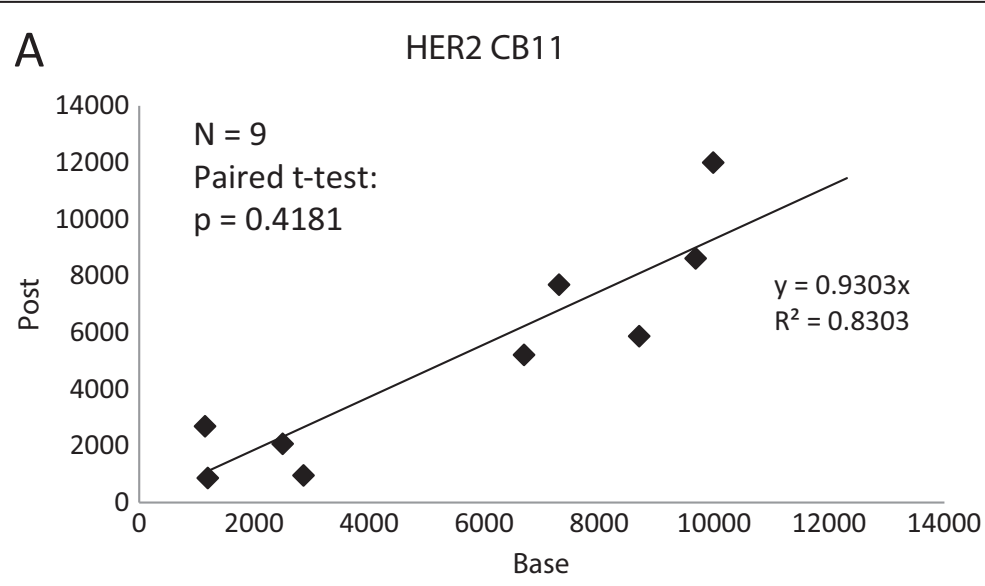

B

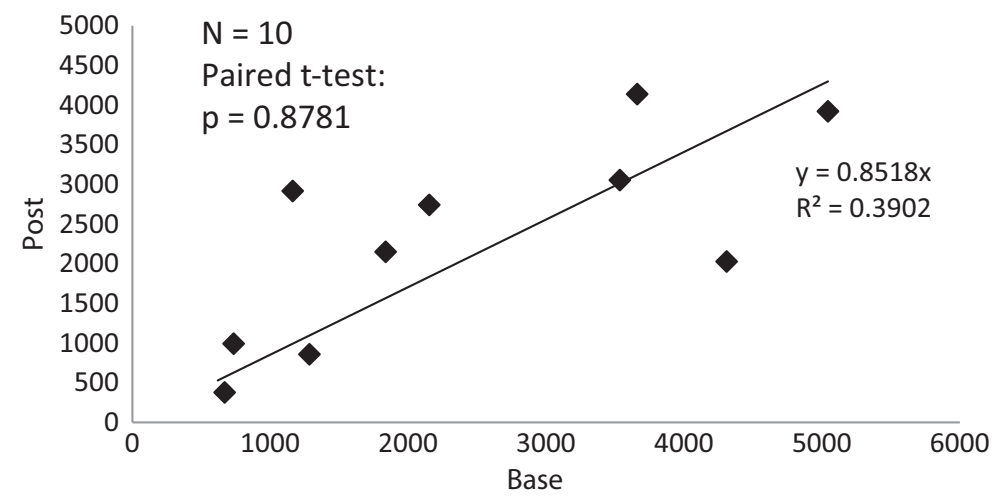

Figure 3 Biomarker change with one dose of trastuzumab. A: HER2 expression level change before (base) and after (post) one dose of trastuzumab shown in AQUA scores; B: phospho HER2 expression level change before (base) and after (post) one dose of trastuzumab shown in AQUA scores. 
In the pHER2 evaluable subset, 10 out of 17 trastuzumab run-in treated patients had paired evaluable baseline and post-treatment biopsies. When we compared the pHER2 level in these 10 pairs, linear regression with a fixed intercept term showed an $\mathrm{R}^{2}$ value of 0.39 and a slope term of 0.85 (post-treatment AQUA score unit as dependent variable). The slope is not statistically significantly different from $1 \quad(\mathrm{p}=0.212)$, suggesting that pHER2 level is not altered by the single dose of trastuzumab treatment. This result is confirmed by the paired t-test of the 9 pairs of baseline and post-treatment biopsies - the mean of post baseline difference is 61.3 AQUA score units $(\mathrm{p}=0.8781)$ (Figure 3B).

\section{No association between HER2 and pHER2 (pY1248)}

We also examined the relationship between HER2 and pHER2 (pY1248) levels. Among baseline biopsies, 21 samples had both evaluable tissues for HER2 and pHER2, and among post-treatment biopsies, 15 samples had evaluable tissues for both HER2 and pHER2. We did not observe an association between HER2 and pHER2 in either baseline biopsies or in post-treatment biopsies (Figure 4).

\section{Discussion}

Few biomarker studies have been able to predict the clinical benefit of the addition of trastuzumab to chemotherapy in the pre-surgical setting, partially because the concept of adding trastuzumab is relatively recent. In addition, patients undergoing pre-surgical therapy typically have limited tissue available for biomarker studies. As a result, studies on pre-surgical therapy tissue are challenging. Furthermore, tissues from the initial core biopsies are small, and often partially or completely exhausted by the diagnostic process. Therefore the availability of tissue for biomarker research in the presurgical setting represents a challenge. In order to obtain more tissue, this study was designed to obtain tissue specifically for research by core biopsy at both the initial time point and 10 days after "run in" therapy. This design allowed assessment of biological changes after one dose pre-surgical treatment. While the study is not large, the dedicated tissue collection allowed us to demonstrate that the level of HER2 is higher in patients that respond, and that the levels do not change between the initial biopsy and the post-treatment biopsy.

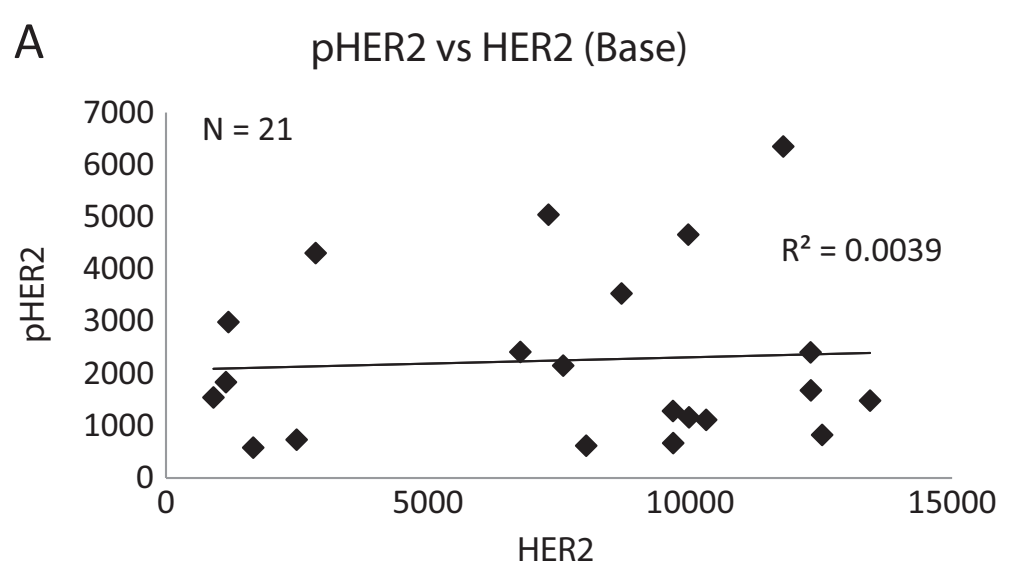

B pHER2 vs HER2 (Post)

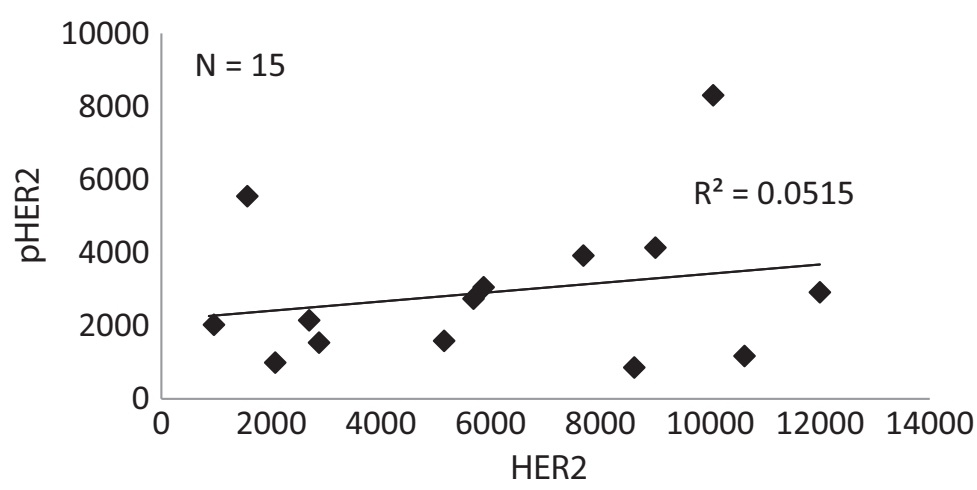

Figure 4 Association between HER2 and pHER2 level. A: Association between HER2 and pHER2 level in baseline biopsies, by AQUA score; B: Association between HER2 and pHER2 level in post "run-in" treatment biopsies by AQUA score. 
Biomarker studies have found several biomarkers, such as HER2 level [23] and PI3Kinase Akt pathway activity $[24,25]$, that are associated with response to trastuzumab in adjuvant and metastatic settings. Consistent with that data, in this study, we were able to demonstrate that the higher level of quantitatively measured HER2 is associated with pathCR. However, we did not find an association between pHER2 (pY1248) level and outcome. Increased phospho-HER2 (pY1248) levels have been associated with worse prognosis $[26,27]$, but this data has been hard to confirm due to challenges associated with phospho-epitope stability. In human tumors, the association between the level of phospho-HER2 and outcome remains controversial $[28,29]$. While our cohort is small, we find no evidence for an association between phospho-HER2 and response to trastuzumab in the pre-surgical setting.

Measurement of phospho-HER2 is challenging since phospho-epitopes are known to be substantially more susceptible to degradation as a function of time to fixation [30,31]. Our recent work (Neumiester et al., in press) and that of others [32] has shown that rapid fixation, as can be achieved by core needle biopsy, is the most effective way to preserve phospho-epitopes. In this study, dedicated core needle biopsy tissues were fixed immediately to minimize artifacts associated with preanalytic cold ischemic time. Although some previous studies predicted that phospho-HER2 should be associated with pathway activity and potentially with drug response, we believe the phospho-epitopes measured here were accurately determined and they do not support a relationship with response in the pre-surgical setting.

\section{Conclusions}

Overall, this work should be considered as a pilot or proof of concept study. Although limited by small sample size, our data suggests that careful measurement of HER2 levels could provide increased specificity in selection of patients for trastuzumab therapy. As the number of therapies that target the HER2 signaling pathway increases it may be important to select patients less likely to respond to trastuzumab so they can be studied with alternative or combination therapies. Future studies that quantify the levels of HER2 in the pre-surgical setting are needed to confirm this observation.

\footnotetext{
Abbreviations

ADCC: Antibody-dependent cell-mediated cytotoxicity; FISH: Fluorescence in situ hybridization; IHC: Immunohistochemistry; OLS: Ordinary least square; OS: Overall survival; PathCR: Pathological complete response; ROI: Region of interest.
}

\section{Competing interests}

D. Rimm declares a potential conflict of interest since he serves as a consultant to Genoptix, Novaritis and Perkin Elmer. W. Sikov has served on advisory boards for Celgene (on which the study sponsor, Abraxis BioScience, Inc., is now a wholly owned subsidiary). M. Abu-Khalaf, L. Harris, N. Sinclair, H. Cheng, Y. Bai, and V. Bossuyt have no competing interests.

\section{Authors' contributions}

$\mathrm{HC}$ did the quantitative measurements in the lab and the data analysis and wrote the first draft of the manuscript. YB assisted in the quantification of the specimens. WS designed the trial, dealt with clinical issues and problems during the conduct of the trial, oversaw clinical data collection and analysis, and assisted in manuscript preparation. NS assisted with the trial execution at the Brown site, overall clinical data collection and analysis, and assisted in manuscript preparation. VB read the pathology at Yale and did all aspects of the work related to determination of pathologic complete response. MK assisted with the trial execution at the Yale site. LH ran the trial at the Yale site, assisted with data analysis and assisted in manuscript preparation. DR runs the lab in which the work was done, reviewed all of the data collection and analysis and assisted in manuscript preparation. All authors read and approved the final version of the manuscript.

\section{Acknowledgements}

The authors would like to recognize the efforts of Lori Charette and her group at Yale Pathology Tissue Services for histology services. This work was supported by NIH RO-1 CA-139431 (DLR and LH) and clinical trial funding from Abraxis BioScience (now a subsidiary of Celgene Corporation).

\section{Financial support}

NIH RO-1 CA-139431 and clinical trial funding from Abraxis BioScience (now a subsidiary of Celgene Corporation).

\section{Author details}

'Department of Pathology, Yale University School of Medicine, 310 Cedar Street, PO Box 208023, New Haven, CT 06520-8023, USA. 'Department of Internal Medicine, Warren Alpert Medical School of Brown University, Providence, RI 02903, USA. ' ${ }^{3}$ Department of Internal Medicine, Yale University School of Medicine, New Haven, CT 06510, USA. ${ }^{4}$ Division of Hematology and Oncology, Case Western Reserve School of Medicine, Cleveland, $\mathrm{OH}$ 44106, USA.

Received: 15 August 2013 Accepted: 2 May 2014

Published: 8 May 2014

\section{References}

1. Slamon DJ, Leyland-Jones B, Shak S, Fuchs H, Paton V, Bajamonde A, Fleming T, Eiermann W, Wolter J, Pegram M, Baselga J, Norton L: Use of chemotherapy plus a monoclonal antibody against HER2 for metastatic breast cancer that overexpresses HER2. N Engl J Med 2001, 344(11):783-792.

2. Yu D, Hung MC: Overexpression of ErbB2 in cancer and ErbB2-targeting strategies. Oncogene 2000, 19(53):6115-6121.

3. Ross JS, Slodkowska EA, Symmans WF, Pusztai L, Ravdin PM, Hortobagyi GN: The HER-2 receptor and breast cancer: ten years of targeted anti-HER-2 therapy and personalized medicine. Oncologist 2009, 14(4):320-368.

4. Cobleigh MA, Vogel CL, Tripathy D, Robert NJ, Scholl S, Fehrenbacher L, Wolter JM, Paton V, Shak S, Lieberman G, Slamon DJ: Multinational study of the efficacy and safety of humanized anti-HER2 monoclonal antibody in women who have HER2-overexpressing metastatic breast cancer that has progressed after chemotherapy for metastatic disease. J Clin Oncol 1999, 17(9):2639-2648.

5. Piccart-Gebhart MJ, Procter M, Leyland-Jones B, Goldhirsch A, Untch M, Smith I, Gianni L, Baselga J, Bell R, Jackisch C, Cameron D, Dowsett M, Barrios CH, Steger G, Huang CS, Andersson M, Inbar M, Lichinitser M, Lang I, Nitz U, Iwata H, Thomssen C, Lohrisch C, Suter TM, Ruschoff J, Suto T, Greatorex V, Ward C, Straehle C, McFadden E, et al: Trastuzumab after adjuvant chemotherapy in HER2-positive breast cancer. N Engl I Med 2005, 353(16):1659-1672.

6. Romond EH, Perez EA, Bryant J, Suman VJ, Geyer CE Jr, Davidson NE, Tan-Chiu E, Martino S, Paik S, Kaufman PA, Swain SM, Pisansky TM, Fehrenbacher L, Kutteh LA, Vogel VG, Visscher DW, Yothers G, Jenkins RB, Brown AM, Dakhil SR, Mamounas EP, Lingle WL, Klein PM, Ingle JN, Wolmark N: Trastuzumab plus adjuvant chemotherapy for operable HER2-positive breast cancer. N Engl J Med 2005, 353(16):1673-1684.

7. Junttila TT, Akita RW, Parsons K, Fields C, Lewis Phillips GD, Friedman LS, Sampath D, Sliwkowski MX: Ligand-independent HER2/HER3/PI3K complex is disrupted by trastuzumab and is effectively inhibited by the PI3K inhibitor GDC-0941. Cancer Cell 2009, 15(5):429-440. 
8. Clynes RA, Towers TL, Presta LG, Ravetch JV: Inhibitory Fc receptors modulate in vivo cytoxicity against tumor targets. Nat Med 2000, 6(4):443-446.

9. Molina MA, Codony-Servat J, Albanell J, Rojo F, Arribas J, Baselga J: Trastuzumab (herceptin), a humanized anti-Her2 receptor monoclonal antibody, inhibits basal and activated Her2 ectodomain cleavage in breast cancer cells. Cancer Res 2001, 61(12):4744-4749.

10. Burstein HJ, Harris LN, Gelman R, Lester SC, Nunes RA, Kaelin CM, Parker LM, Ellisen LW, Kuter I, Gadd MA, Christian RL, Kennedy PR, Borges VF, Bunnell CA, Younger J, Smith BL, Winer EP: Preoperative therapy with trastuzumab and paclitaxel followed by sequential adjuvant doxorubicin/cyclophosphamide for HER2 overexpressing stage II or III breast cancer: a pilot study. J Clin Oncol 2003, 21(1):46-53.

11. Coudert BP, Arnould L, Moreau L, Chollet P, Weber B, Vanlemmens L, Molucon C, Tubiana N, Causeret S, Misset JL, Feutray S, Mery-Mignard D, Garnier J, Fumoleau P: Pre-operative systemic (neo-adjuvant) therapy with trastuzumab and docetaxel for HER2-overexpressing stage II or III breast cancer: results of a multicenter phase II trial. Ann Oncol 2006, 17(3):409-414

12. Van Pelt AE, Mohsin S, Elledge RM, Hilsenbeck SG, Gutierrez MC, Lucci A Jr, Kalidas M, Granchi T, Scott BG, Allred DC, Chang JC: Neoadjuvant trastuzumab and docetaxel in breast cancer: preliminary results. Clin Breast Cancer 2003, 4(5):348-353.

13. Hurley J, Doliny P, Reis I, Silva O, Gomez-Fernandez C, Velez P, Pauletti G, Powell JE, Pegram MD, Slamon DJ: Docetaxel, cisplatin, and trastuzumab as primary systemic therapy for human epidermal growth factor receptor 2-positive locally advanced breast cancer. J Clin Oncol 2006, 24(12):1831-1838.

14. Limentani SA, Brufsky AM, Erban JK, Jahanzeb M, Lewis D: Phase II study of neoadjuvant docetaxel, vinorelbine, and trastuzumab followed by surgery and adjuvant doxorubicin plus cyclophosphamide in women with human epidermal growth factor receptor 2-overexpressing locally advanced breast cancer. J Clin Oncol 2007, 25(10):1232-1238.

15. Buzdar AU, Ibrahim NK, Francis D, Booser DJ, Thomas ES, Theriault RL, Pusztai L, Green MC, Arun BK, Giordano SH, Cristofanilli M, Frye DK, Smith TL, Hunt KK, Singletary SE, Sahin AA, Ewer MS, Buchholz TA, Berry D, Hortobagyi GN: Significantly higher pathologic complete remission rate after neoadjuvant therapy with trastuzumab, paclitaxel, and epirubicin chemotherapy: results of a randomized trial in human epidermal growth factor receptor 2-positive operable breast cancer. J Clin Oncol 2005, 23(16):3676-3685.

16. Gianni L, Eiermann W, Semiglazov V, Manikhas A, Lluch A, Tjulandin S, Zambetti M, Vazquez F, Byakhow M, Lichinitser M, Climent MA, Ciruelos E, Ojeda B, Mansutti M, Bozhok A, Baronio R, Feyereislova A, Barton C, Valagussa P, Baselga J: Neoadjuvant chemotherapy with trastuzumab followed by adjuvant trastuzumab versus neoadjuvant chemotherapy alone, in patients with HER2-positive locally advanced breast cancer (the NOAH trial): a randomised controlled superiority trial with a parallel HER2-negative cohort. Lancet 2010, 375(9712):377-384.

17. Untch M, Rezai M, Loibl S, Fasching PA, Huober J, Tesch H, Bauerfeind I, Hilfrich J, Eidtmann H, Gerber B, Hanusch C, Kuhn T, du Bois A, Blohmer JU, Thomssen C, Dan Costa S, Jackisch C, Kaufmann M, Mehta K, von Minckwitz G: Neoadjuvant treatment with trastuzumab in HER2-positive breast cancer: results from the GeparQuattro study. J Clin Oncol 2010, 28(12):2024-2031.

18. Andre F, Mazouni C, Liedtke C, Kau SW, Frye D, Green M, Gonzalez-Angulo AM, Symmans WF, Hortobagyi GN, Pusztai L: HER2 expression and efficacy of preoperative paclitaxel/FAC chemotherapy in breast cancer. Breast Cancer Res Treat 2008, 108(2):183-190.

19. Denkert C, Huober J, Loibl S, Prinzler J, Kronenwett R, Darb-Esfahani S, Brase JC, Solbach C, Mehta K, Fasching PA, Sinn BV, Engels K, Reinisch M, Hansmann ML, Tesch $\mathrm{H}$, von Minckwitz G, Untch M: HER2 and ESR1 mRNA expression levels and response to neoadjuvant trastuzumab plus chemotherapy in patients with primary breast cancer. Breast Cancer Res 2013, 15(1):R11.

20. Camp RL, Chung GG, Rimm DL: Automated subcellular localization and quantification of protein expression in tissue microarrays. Nat Med 2002 8(11):1323-1327

21. Gustavson MD, Bourke-Martin B, Reilly DM, Cregger M, Williams C, Tedeschi G, Pinard R, Christiansen J: Development of an unsupervised pixel-based clustering algorithm for compartmentalization of immunohistochemical expression using automated quantitative analysis. Appl Immunohistochem Mol Morphol 2009, 17(4):329-337.

22. Giltnane JM, Molinaro A, Cheng H, Robinson A, Turbin D, Gelmon K, Huntsman D, Rimm DL: Comparison of quantitative immunofluorescence with conventional methods for HER2/neu testing with respect to response to trastuzumab therapy in metastatic breast cancer. Arch Pathol Lab Med 2008, 132(10):1635-1647.

23. Piccart M, Lohrisch C, Di Leo A, Larsimont D: The predictive value of HER2 in breast cancer. Oncology 2001, 61(Suppl 2):73-82.

24. Nagata Y, Lan KH, Zhou X, Tan M, Esteva FJ, Sahin AA, Klos KS, Li P, Monia BP, Nguyen NT, Hortobagyi GN, Hung MC, Yu D: PTEN activation contributes to tumor inhibition by trastuzumab, and loss of PTEN predicts trastuzumab resistance in patients. Cancer Cell 2004, 6(2):117-127.

25. Park BH, Davidson NE: PI3 kinase activation and response to trastuzumab therapy: what's neu with herceptin resistance? Cancer Cell 2007, 12(4):297-299.

26. Thor AD, Liu S, Edgerton S, Moore D 2nd, Kasowitz KM, Benz CC, Stern DF, DiGiovanna MP: Activation (Tyrosine phosphorylation) of ErbB-2 (HER-2/neu): a study of incidence and correlation with outcome in breast cancer. J Clin Oncol 2000, 18(18):3230-3239.

27. DiGiovanna MP, Stern DF, Edgerton SM, Whalen SG, Moore D 2nd, Thor AD: Relationship of epidermal growth factor receptor expression to ErbB-2 signaling activity and prognosis in breast cancer patients. J Clin Oncol 2005, 23(6):1152-1160.

28. Hudelist G, Kostler WJ, Attems J, Czerwenka K, Muller R, Manavi M, Steger GG, Kubista E, Zielinski CC, Singer CF: Her-2/neu-triggered intracellular tyrosine kinase activation: in vivo relevance of ligand-independent activation mechanisms and impact upon the efficacy of trastuzumab-based treatment. Br J Cancer 2003, 89(6):983-991.

29. Jensen JD, Knoop A, Laenkholm AV, Grauslund M, Jensen MB, Santoni-Rugiu E, Andersson M, Ewertz M: PIK3CA mutations, PTEN, and pHER2 expression and impact on outcome in HER2-positive early-stage breast cancer patients treated with adjuvant chemotherapy and trastuzumab. Ann Oncol 2012, 23(8):2034-42.

30. Bai Y, Tolles J, Cheng H, Siddiqui S, Gopinath A, Pectasides E, Camp RL, Rimm DL, Molinaro AM: Quantitative assessment shows loss of antigenic epitopes as a function of pre-analytic variables. Lab Invest 2011, 91(8):1253-1261

31. Pinhel IF, Macneill FA, Hills MJ, Salter J, Detre S, A'Hern R, Nerurkar A, Osin P, Smith IE, Dowsett M: Extreme loss of immunoreactive p-Akt and p-Erk1/2 during routine fixation of primary breast cancer. Breast Cancer Res 2010, 12(5):R76.

32. Yildiz-Aktas IZ, Dabbs DJ, Bhargava R: The effect of cold ischemic time on the immunohistochemical evaluation of estrogen receptor, progesterone receptor, and HER2 expression in invasive breast carcinoma. Mod Pathol 2012

doi:10.1186/1471-2407-14-326

Cite this article as: Cheng et al:: Quantitative measurements of HER2 and phospho-HER2 expression: correlation with pathologic response to neoadjuvant chemotherapy and trastuzumab. BMC Cancer 2014 14:326.

\section{Submit your next manuscript to BioMed Central and take full advantage of:}

- Convenient online submission

- Thorough peer review

- No space constraints or color figure charges

- Immediate publication on acceptance

- Inclusion in PubMed, CAS, Scopus and Google Scholar

- Research which is freely available for redistribution 